\title{
Technical Research On CAN Bus Software Decode And Performance Index Measurement Of Inter-Frame Space
}

\author{
Huiming $\mathrm{Wu}^{1}$, Ling $\mathrm{Li}^{2}, \mathrm{Hao} \mathrm{Li}^{3}$ \\ Academy of Armored Forces Engineering, Beijing \\ 100072, China.
}

Keywords: CAN bus, Software decode, Inter-frame space.

\begin{abstract}
In order to monitor CAN bus device and network real-time and effectively, performance index scheme on CAN bus software decode as well as precisely measure performance index CAN bus inter-frame space is designed by the method of collecting physical layer wave form directly, so as to provide new solving method for CAN bus performance test, fault diagnose as well as location.
\end{abstract}

\section{INTRODUCTION}

CAN (Control Area Network) is one of the most extensive application fieldbus worldwide, which had launched by Germany Bosh company initially. It is a kind of serial data communication bus which is extensively applied in vehicle to exchange data between inner measure and execution unit. As non-destructive arbitration technique is adopted, CAN bus possess higher reliability. The CAN bus direct communication maximum range is $10 \mathrm{~km}$, highest communication speed is $1 \mathrm{MB} / \mathrm{s}$.

CAN bus control network already applied in areas of vehicle, aerospace and military equipment. As CAN bus transmission data involve those factors which include real-time collection of vehicle control system, as well as operating state, setup parameter and running instruction of integrated vehicle, components and parts, therefore, the research on vehicular CAN performance testing method is necessary apparently.

The current testing equipment adopted the design of bus interface chip (module). What the data frames this kind of testing equipment obtained is incomplete, as the lack of time information. Therefore, the interrelation of data frame transmission among multiple bus cannot be achieved. Hence, to design another kind of testing scheme which can transmit data frame independent of bus interface chip (module) is necessary. This scheme involves the technical index on bus inter-frame space which is measuring by following methods, directly collecting data transmission waveform on physical layer, decoding software and so on.

\section{GENERAL SCHEME}

At present, all kinds of special protocol chips are adopted while CAN bus testing, only get intact data frame after decode, not cover the actual whole transmission data running in bus(include incorrect frame). The incorrect data frames include incorrect bit and verify which are caused by distortion and interference in bus transmission wave form data are filtered by special bus protocol chips. Actually, those filtered data are vital to test data transmission network performance and fault location. According to them, fault point can be located. At present, fault location couldn't find out depend on those abnormal data, because of the testing devices filter those abnormal data frame.

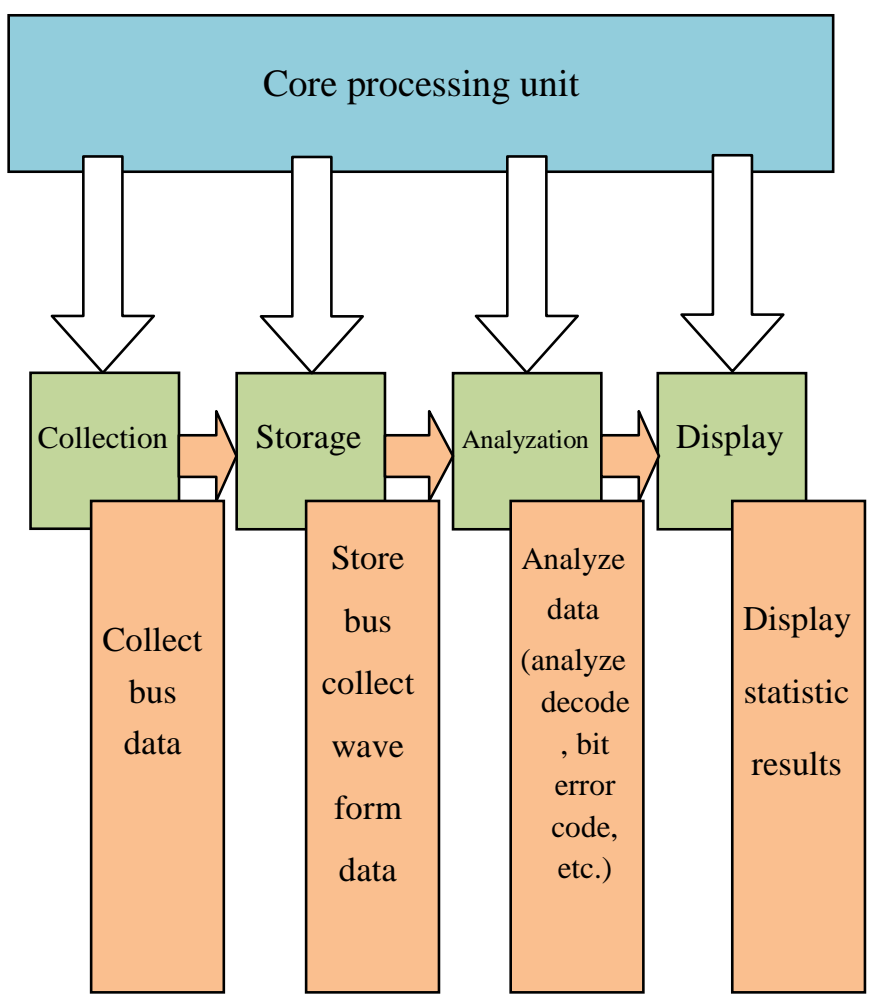

Fig. 1. System processing flow diagram 
Since there is no uniformly time reference for the bus data frame information which was obtained by corresponding bus special protocol chips, thus what collected from the corresponding bus special protocol chips are only the sequential order but exactly time information. Therefore, the measurement of inter-frame space between bus command frame and response frame cannot achieve. Especially, in the comprehensive analysis on multiple bus data frames, it cannot be taken into account while judging working performance of system, as lacking of associated time information.

The author adopts the method of translating the collective physical layer wave data to acquire data of transmission frame, to decode and analyze data frame with software. The exact time of data frame arrived on transmission line are clear, on account of adopted timer collecting data. Therefore, time difference between frames can be calculated exactly, and to obtain technical index on responsive time. Finally, we could to provide the new testing method for bus device and network test as well as the basis for fault location and diagnose. The system processing flow is show as Fig 1.

\section{CAN BUS SOFTWARE DECODE TECHNOLOGY}

At present, the vehicular bus test is finished by operating special interface protocols chips which are coupled types to the bus. However, to analyze data frame by software should acquiring transmission frame data by translated physical layer data wave. The work of obtaining data frame directly from collective wave data could finished unless some key theologies were tackled, such as initial time of data frame location, measure pulse width, automatic transmission code rate calculation and analyze data frame decode on the basis of CAN protocol etc.

The key technology of software decode bus data frame are following, firstly, to calculate pulse width of wave data which were collected from high speed data; Secondly, to obtain baud rate of vehicular bus transmission data; thirdly, to acquire the starting point of frame from collected wave, fourthly, resulted corresponded data by translate wave decode according to coupling bus frame format, finally, to display waveform and translated date by software for late testing. As well as the following works, to deal with the effective factors while calculated baud rate, such as the interference from random pulse wave, converting collective wave data to CAN data frame immediately, decoding the integrated data frame (include error frame), in order to provide reliable frame data for later analyze error frame and acquire exactly error frame quantity, finally providing basis for bus performance evaluation and fault location.

Operating software to transcode should to calculate pulse width of wave data which collected from high speed data. It include those steps, obtaining baud rate of bus transmission data, acquiring the starting point of frame from collective waveform, coveting code according to tested bus data protocol and transcoding wave code ,resulting corresponded data and displaying data frame results. The software decode operation process is show as Fig 2. Software interface of waveform decode bus data is show as Fig3. Decode result is show as Fig 4.

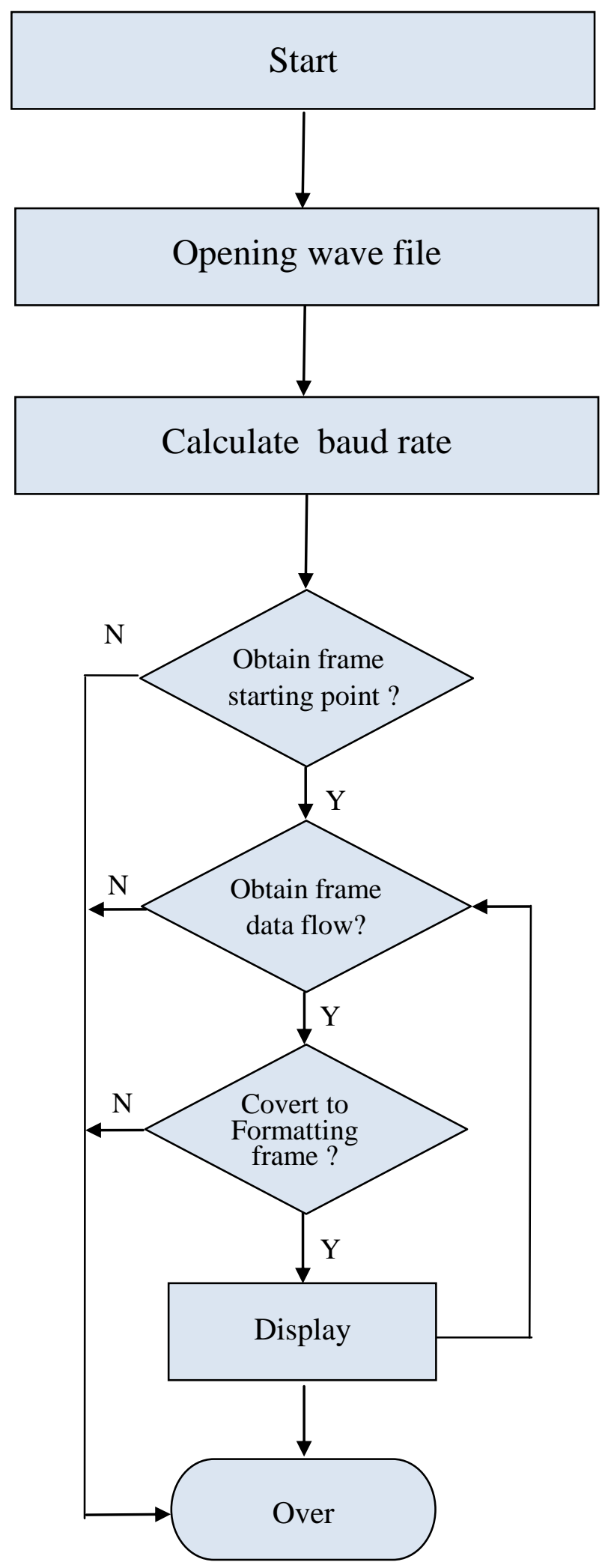

Fig 2 Data frame decode process diagram 
The average minimum-width method is adopted in automatic calculation of bus data frame pulse width and transmission code rate. The transmission rate of this bus can be result by statistically calculated frame pulse width. According to corresponding bus protocol, double-threshold method is adopted while setting start time of the bus data frame, so as to make comprehensive discretion depending on start pulse feature and frame header information. According to responding bus protocol, the software decode of data frame is based on transforming the format of data frame from pulse waveform to data bits characters 0,1 bit-by-bit, finally, interpreting frame date. After decoding process is finished, to calibrate and to storage exactly time-stamp of data frame on the data frame start time. Hence, the precision time-stamp could be microsecond level. Both the data frame transmission waveform and the results of data frame which decoded by software could display on viewing screen at the same times. The accurately measure work on inter-frame space of two channels data frames also can completed at the same times. By account on inter-frame space data of data frame, not only the transmission dense region can be easily located, but also the congestion time can be quickly located.

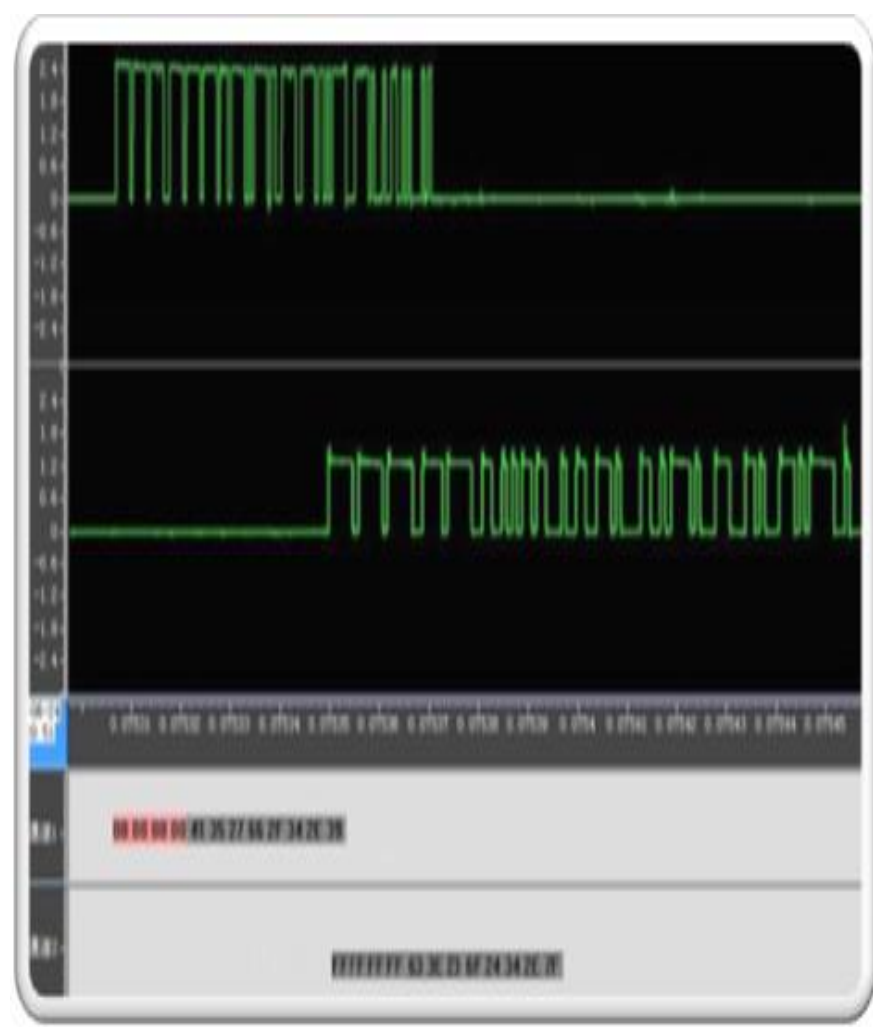

Fig 3 Bus data waveform

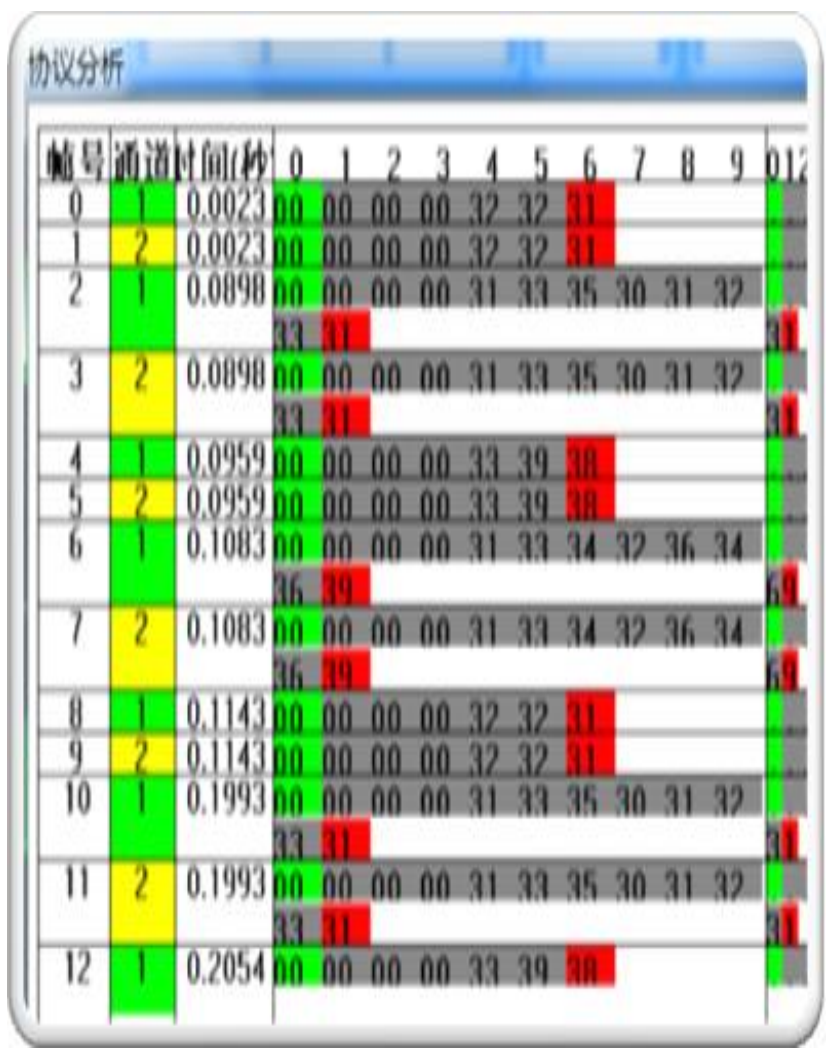

Fig 4 Decode data of bus waveform

\section{EXACT MEASURE ON INTER-FRAME SPACE INDEX}

As the CAN bus, there is strictly standard on time between frames, the sending time and responding time of frame must be acquired exactly. In the past, bus interface chip is adopted in obtaining bus data frame, but there is no timing circuit on interface chip, this index cannot be measured as the time is estimated.

Our test device adopt the method to acquire command frame by directly collect waveform at psychical layer, arrived time of data frame can be exactly got on the transmission line, as the timing is employed in collecting data. Therefore, the time difference between commanding data frame and responding data frame can be calculated exactly, the index on respond time is deduced.

Interval-time index exactly measure method is to mark timestamp on each data frame transmit in bus, that is to make signal on data waveform which transmission on bus from frame or information along the time, then to collect data frame waveform and to mark each frame data with time-tag while analyzing. The processes are below:

The system starts to collect data waveforms which transmit on bus and storing them into large-capacity storage device. In storage thread, mark each sample with "time-tag" which is 
affixed system time. Therefore, the start time, end time and the lasting time of each sample on waveform are constant, but also consistent with time of integrated testing system.

At the same time to acquire data flow, the time of sample are also acquired, while transcode waveform. The calculated method is below: Add the time migration $\Delta$ t between sample and starting time of sample with the starting time of sample which is the "time-tag" of data flow. Put the "time-tag" at the front of frame ID, while display decode, time format is hour/minute/second/millisecond/microsecond. Distributing in five groups of 16 bits separately, resolution not less than 1 microsecond.

Therefore, the measure accuracy could reach 1 microsecond as sample rate is $10 \mathrm{Msa} / \mathrm{s}$. See it in Fig 5.

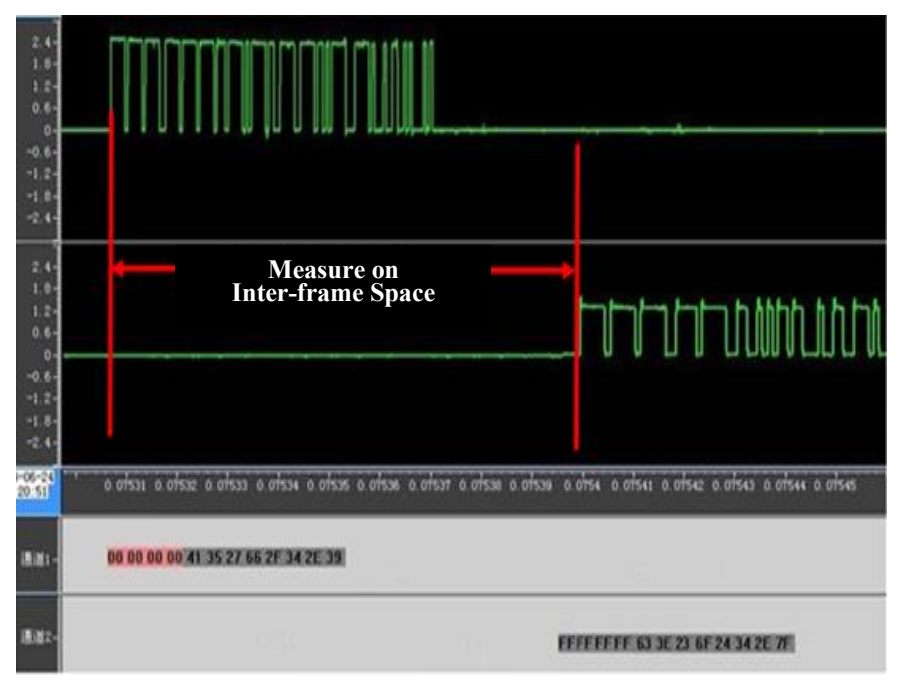

Fig 5. Time relational graph of each route data frame

Taking the time-tag as an example, it is added to CAN bus message inter-frame, and use to illustrate time testing process of sampling in frame or massage, as it shows in fig.6. It is clear in the figure that the time migration $\Delta \mathrm{t} 1$ of frame 1 collecting time which is relative to the start collecting time is
$0.06076 \mathrm{~s}$, means $60.76 \mathrm{~ms}$. The time migration $\Delta \mathrm{t} 2$ of frame 2 collecting time which is relative to the start collecting time is $0.061 \mathrm{~s}$, means $61 \mathrm{~ms}$.Using $\Delta \mathrm{t} 2$ minus $\Delta \mathrm{t} 1$, resulted interframe between frame 1 and frame 2 , which is $0.24 \mathrm{~ms}$, means $240 \mu \mathrm{s}$. This result illustrates that the testing system which is designed in this thesis, could achieve inter-frame testing on adjacent message frames.

\section{CONCLUSION}

The abnormal data frame and its occurrence time can be located based on the collective wave data. According to this data, this abnormal frame also can be located which lay the foundation for system fault diagnosis. This online measure method could found abnormal in bus transmit signal frame. By taking advantage exclusive method, we can located the exact device which send abnormal bus wave signal, found out unmatched route on bus, calculated delay between commanding frame and responsive frame exactly, acquired measure of frame delay technical index and measure bit error rate of bus transmission and so on. Consequently, it provide a new kind of resolving way for CAN bus performance testing, fault diagnose and location.

\section{References}

[1] Zhang Zhongxiong, Wang Bo, "The Application of CAN Communication Network in Automobile Comprehensive Performance Measuring" J MPU pp.2728,2002(2).

[2] Shi Jiugen, Zhang Peiren, Cheng Zhenyong. "The Design Technology of CAN Fieldbus System." M National Defense Industry Press 2004.

[3] Zhu Qidan, Wang Tong. "The Modeling and Simulation on CAN Communication Physical layer". J Computer Engineering and Applications pp.11-15,2008,44(36).

[4] Rao Yuntao, Zou Jijun. "The Principle and Applied Technology of Fieldbus CAN". M Beijing: Beijing University of Aeronautics and Astronautics Press, 2007.

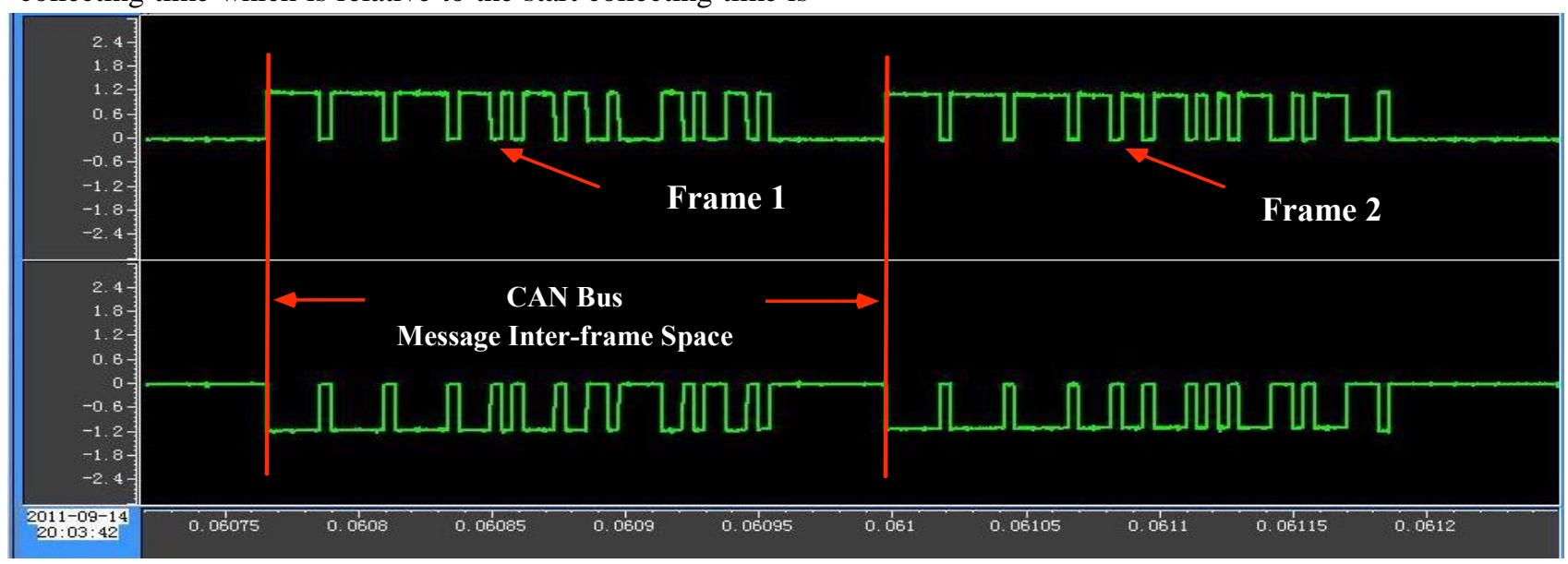

Fig 6. Sketch map of CAN bus message inter-frame space testing 